\title{
Improvement of the traffic safety system
}

\author{
Ksenia Shubenkova ${ }^{1 *}$, Aleksey Boyko ${ }^{1}$, Gulnara Yakupova ${ }^{1}$, and Kirill Magdin ${ }^{1}$ \\ ${ }^{1}$ Kazan Federal University, 423812 Naberezhnye Chelny, Russia
}

\begin{abstract}
Increase of the road safety is the main goal in the sphere of transport management. Authors state that the decision-making process should be based on the analysis of statistical data on the violations, traffic reasons of accidents and problem areas. Official statistics of the State Traffic Safety Inspectorate is used as an initial information. As a method to determine factors influencing the traffic safety, the so-called Haddon matrix was used. Efficiency of the proposed recommendations is analyzed from the viewpoint of their role in the process of ensuring safety and sustainability of the city transport system.
\end{abstract}

\section{Introduction}

The processes of urbanization and globalization of economics are accompanied by an increase in the need for cargo and population transportation. Rapid growth of motorization and traffic volumes causes reduction of the sustainability and safety of transport system that is confirmed by negative impact on the environment and increase in the number of road traffic injuries. According to the "Global status report on road safety 2015", despite road safety improvement, there are still about 1.25 million people who die each year as a result of traffic accidents. In recognition of the scale of this health and development problem - and the possibility to have a positive impact on it - the Decade of Action for Road Safety (20112020) was established.

Many countries are successful in achieving and sustaining reductions in death rates as a result of accidents. This has been made possible by improving, monitoring and enforcing national laws, as well as increasing road and vehicle safety. However, there is a significant gap between high-income and low-and-middle-income countries: $90 \%$ of road traffic deaths occur in the countries that use just $54 \%$ of the world's vehicles. Almost half (49\%) of all deaths on the roads around the world are among the most vulnerable road users motorcyclists, pedestrians and cyclists. System approach to ensuring road safety together with rational combination of technical and managerial interventions, allow preventing most of the accidents. There is a considerable evidence on interventions that are effective in making roads safer: many of high income countries in the European Region are successful at reducing deaths and injuries on roads even despite increasing motorization [5].

* Corresponding author: ksenia.shubenkova@gmail.com 


\section{Problem Description and Research Objectives}

Compared with European countries, the level of motorization in Russia is not so high, but it is rapidly growing. The country suffers from problems caused by high percentage of individual transport in the total traffic volume, such as: congestion, air pollution, noise, energy consumption and road accidents [7]. These issues are noted in Russian Federal Target Program [3] as priority problems to be solved. The efforts that have been already made are successful: despite the growing motorization level, the number of road accidents and the severity of their consequences have been decreasing over the past five years. Fig. 1 shows that traffic accidents' rate is still high in Russia therefore causes of accidents and the choice of adequate actions to decrease their number have to be analyzed carefully.

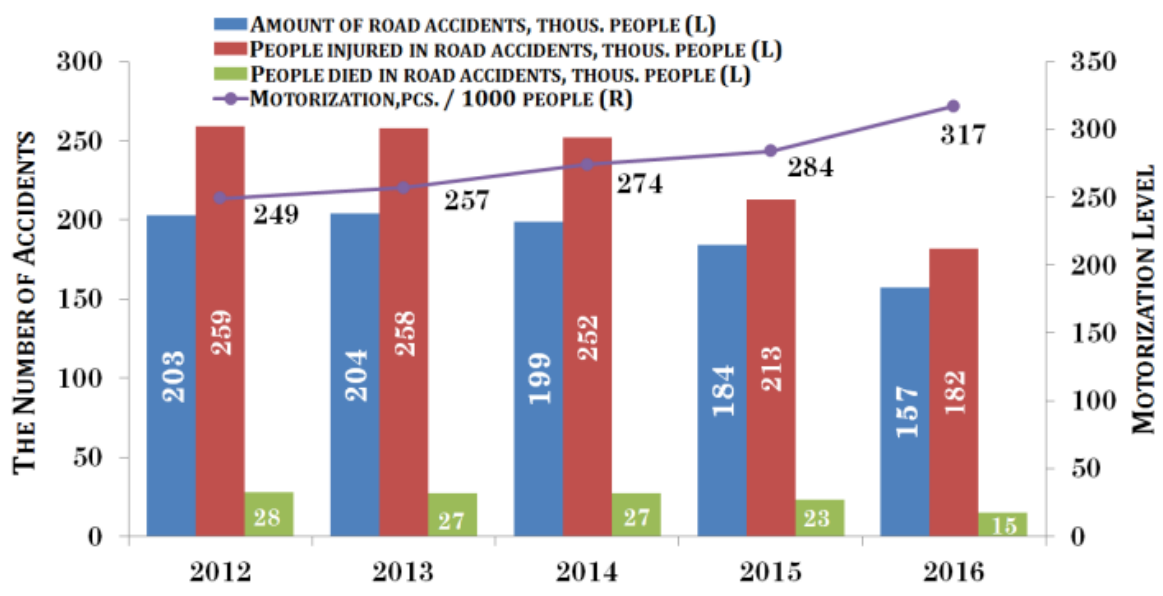

Fig. 1. Dynamics of motorization, statistics of road accidents and their consequences

The most common causes of deaths in road traffic accidents in 2015 used to be: head-on or lateral collision - 32754 people injured in accidents; poor quality of road pavements - 30 667 people injured in incidents, with about 4000 people died; hitting a pedestrian - 23724 cases, including 2731 accidents when people died; vehicle roll-over - 7906 people were injured and 2731 died; faulty state of the vehicle - 945 cases, including 145 accidents when people died.

Russia is on the top place of road mortality rate among European countries. The main causes of the road traffic accidents are:

- Alcoholic intoxication (this causes 40 percent of all road accidents).

- Distracting circumstances. One of the main of them is making a telephone call. Even if a driver talks on a hands-free cell phone while driving it increases the probability of an accident in 3-6 times.

- External objects that can also cause dispersal of concentration and, as a result, a slow reaction and an inability to make the right decision on the road.

- Aggressive driving, that increases the risk of a traffic accident up to $40 \%$.

- Traffic rule violations. Despite the fact that refuse to use seat belts and the child seats is not a direct cause of the accident, it significantly increases the risk of death in case of emergency.

- Tiredness of the driver. This is an insignificant, but, nevertheless, possible cause of the accident. As a result of the long road, a person gets tired, starts to rush, and incorrectly estimates the traffic situation. All of this can lead to the death of the driver, as well as of passengers. 
The objective of this study is to propose ways to improve road safety that should be based on the analysis of the statistics of the traffic rule violations and the implemented actions' efficiency.

\section{Methods}

According to [8], improvement of the system, which manages the activities to increase the road safety, must be realized in three directions: prevention of accident, reducing the severity of accident consequences and post-accident clean-up on a segment of the road network.

\subsection{Ways to prevent road accidents}

There is an active safety of vehicles and infrastructure. An active safety of road includes features, which prevent the appearance of accident, such as, for example, good adherence with road surface, influencing dynamics of vehicle, opportunity to stop before the obstacle, safety level of a given maneuver. The road infrastructure includes the following elements of active safety: geometrical sizes and specifics (for example, roundabouts), existence of roadside, division of transport, bicycle and pedestrian traffics (underground or overhead pedestrian crossings). Such measures as horizontal road marking, road signs, artificial lighting, directed on the prevention of accident occurrence could be considered also as components of active road safety. One for the most important requirements to ensure road safety is traffic intensity's reduction that could be done by efficient organization of traffic flows due to increasing probability of accident's occurrence in the complicated road conditions.

Active safety of vehicle includes elements influencing directly the functions of a vehicle and its performance characteristics while moving. According to researchers [4, 14-16, 17], reducing the role of a human factor in the large-scale system management process will decrease the risk of accidents causing serious consequences in the conditions of large cities and megapolises. To achieve this, advanced technologies are nowadays aimed on the improvement of advanced driver assistance systems (ADAS), using such technologies as $\mathrm{V} 2 \mathrm{X}$ and $5 \mathrm{G}$ to communicate with other road users. Traditional technologies to support driver allow to detect some objects, to carry out a basic classification, to inform driver about dangerous situations and, in some cases, to slow down or stop the movement of vehicle. However, at the same time, if these warnings are poorly designed, they may distract drivers, decelerate their responses, and reduce the road safety. Thus, it is necessary to understand that the introduction of new technical and technological solutions is connected with the occurrence of new problems, which could require new methods and tools to solve them.

The low level of safety on the roads is largely caused by traffic rules violations. The study by Martinussena et al [9] is focused on identification of two aspects: risk groups of drivers and reliable predictors of safe or unsafe driving behavior. The descriptive statistics showed that the percentage of people, who had a registered traffic offense, was higher within the group of "violating unsafe drivers".

\subsection{Traffic Accidents' Consequences Reduction}

Reduction of road traffic injuries during accidents is achieved by a passive safety of vehicles and infrastructure. Elements, which are included in the system of vehicle passive safety, do not influence functions and performance characteristics of a vehicle. They are intended to protect road users in case of accident. To reduce people's injuries, modern mathematical methods and models for better crash energy absorption are used during construction and 
development of vehicle body components. Additionally, the important role to ensure a passive safety for driver and passengers is to use a number of facilities and components, such as airbags, good functional design of seat and steering tube as well as seat belts and child seats. According to World Health Organization, wearing a seat-belt reduces the risk of fatality among drivers and front-seat passengers by $45-50 \%$ and the risk of minor and serious injuries by $20-45 \%$, respectively. Child restraints reduce the likelihood of fatalities in case of an accident by approximately $90 \%$ among infants and between $54 \%$ and $80 \%$ among young children. To decrease injuries of pedestrians, special construction and materials for front bumpers are utilized.

The following measures are understood as a passive road safety: measures directed on the reduction of the severity of accidents' consequences, which appears due to loss of control or vehicle stability; destabilization of vehicle system elements (trailer breaking away, movement of heavy goods in the vehicle boot); unexpected attack of illness, blindness from oncoming headlights in the darkness. To reduce the severity of consequences, which concern collision with an obstacle, exit off the roadside or crossing of the central reserve, safety fences are installed. They prevent a vehicles' exit off the subgrade and as a result of bending and due to the elastic structure deformation of the fence contribute to the vehicles' trajectory change and to minimization of the shock loads. In the USA and countries of Western Europe high monolithic or prefabricated constructions of concrete barriers are often installed on the central reserves and on the edge of the carriageway of the bridge or the highway. Such barriers deflect running-down vehicles and slow down their speed without inflicting damage [10].

A significant decrease in the severity of accidents' consequences can be achieved by reducing speed, maintaining a large safe distance, and refraining from overtaking. Thus, tools of violations registration (cameras registering speed, sensors registering vehicles running the red light, etc.) in conjunction with other elements of transport infrastructure that reduce speed and number of other violations which increase the accidents' consequences severity are also understood as a passive road safety. For example, such infrastructure interventions as speed bumps help to manage speed and reduce the severity of a crash $[7,8]$.

However, when making decision on the infrastructure's change, it should be borne in mind that such measures can also have negative consequences. Thus, in the research [1], it was found out that an air pollution with particulate matter increases by $2-5$ times near the speed bumps, because each vehicle has to slow down to overcome them. In Russia, most of speed bumps are installed before unregulated pedestrian crossings. In some cases, such decision is unjustified, especially in areas with low intensity and non-stationary pedestrian flow. The best decision that does not raise emissions is the use of pedestrian push-button traffic lights in such areas.

\subsection{Relieving the Consequences of Traffic Accidents}

Emergency response systems can help to reduce consequences of injuries by providing prompt medical assistance after the accident. Timely information of road users about accidents occurred on a particular site, will allow drivers to correct their route to bypass the problem area and to avoid the traffic congestion associated with traffic accidents.

An important area in the field of improving road safety is decision-making based on a statistical data analysis. For example, data on traffic violations can be used to create a list of drivers to work with in order to prevent future offenses. Depending on the severity and frequency of drivers' violations, prevention measures can be as follows: preventive conversations and even the withdrawal of the driving license till retaking an exam. Conclusions made in [11] could be used to educate drivers about the risks associated with various traffic rule violations and help drivers adopt safe driving behavior on the roads. In 
the case of regular violations and accidents, occurring at the same site this requires a detailed analysis of the traffic organization in order to reconstruct the infrastructure. Analysis of statistical data collected after the implementation of changes will allow determining effectiveness of the measures undertaken.

\subsection{Initial Data and Results of the Analysis}

Official statistics of the State Traffic Safety Inspectorate (STSI) of the city Naberezhnye Chelny as well as the data from the official website of the STSI of Russian Federation [13] are used as an initial information. Preventive measures to improve the road safety include identification of violations that can be divided into two categories: (1) violations that increase the possibility of an accident (drink-driving, leaving the oncoming traffic) and (2) violations that increase the severity of accident's consequences (disusage of child restraints and seatbelts).

Fig. 2a shows the dynamics of violations in the past three years. In our opinion, decrease in the number of traffic violations in 2015 can be connected to the raise of penalties, because after the law allowing to pay a half of the penalty size in case of payment within 20 days from the date of violation has been adopted, in 2016, there was a growth of violations. Such dynamics is caused by this law that, in fact, canceled the increase of penalties.

Analysis of violations statistics by days of week (Fig. 2b) shows the growth of both violations and accidents at the end of the working week (on Friday). At weekends, the number of violations is still high, but not as high as within the week. This can be explained by reinforced patrolling by STSI's crews at weekends that allows immediate identification of potentially dangerous situations, preventing the accident.

Decline in number of road accidents in recent years caused by over-speeding or discrepancy of the chosen speed to specific road conditions is primarily connected to the active implementation of cameras that capture high-speed mode disturbances. We have compared data on the revealed facts of violations and road accidents caused by the same factors (Fig. 3b). Decrease in the number of violations and accidents at pedestrian crossings is caused by toughening of sanctions for such violations. In addition, speed bumps before non-signalized crosswalks were installed in 2016 that has led to a reduction in traffic flow's speed in this areas and, as a consequence, decreased the possibility of accidents. There also was a decline in number of accidents caused by other reasons.
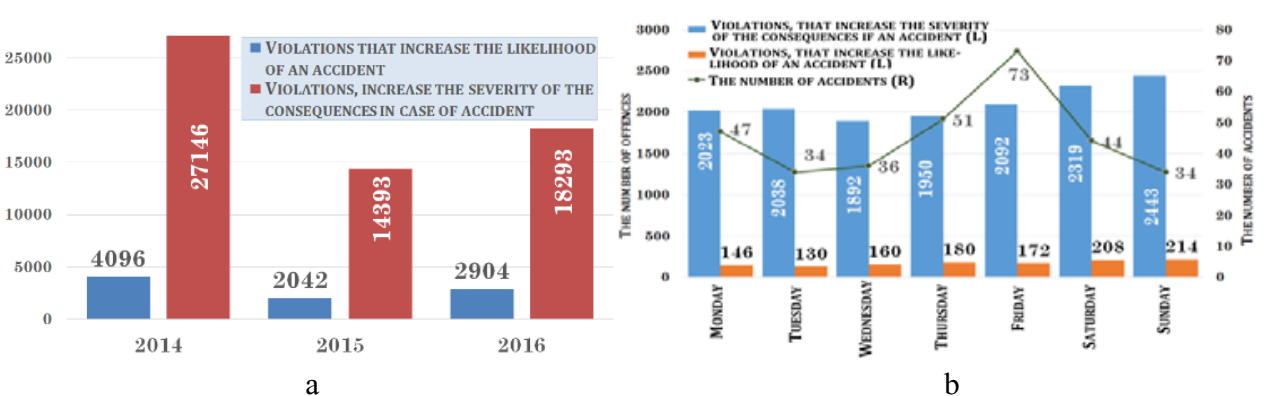

Fig. 2. Dynamics of violations a) by years, b) by days of week.

\section{Results and Discussions}

Improving road safety involves identifying the risk factors that contribute to crashes and injuries, then identifying the interventions that reduce the risks associated with those factors. One of the methods to identify factors related to road accidents is a model, called "Haddon's Matrix", which divides factors into human, vehicular and environmental causes across three 
temporal phases - pre-crash, crash and post-crash $[2,6,12,5]$. We have structured the existing ways to improve road safety by phases in time of the event (Fig. 4).

Once the multiple factors associated with a crash are identified and analyzed, countermeasures can be developed and prioritized for implementation over short-term and long-term periods. The proposed algorithm includes the following steps: (1) analysis of violations and accidents statistics, (2) identification of violations that increase the probability of accident, (3) formation of the list of actions to prevent violations, causing the growth of the accidents' probability (filling the "before crash" column), (4) identification of violations that may deteriorate the severity of accidents, (5) formation of the list of actions to prevent violations, aggravating the severity of accidents (filling the column "in crash"), (6) identification of violations that prevent rapid post-accident clean-up, (7) formation of the list of actions to prevent violations hindering rapid post-accident clean-up (filling the column "after crash").

After the implementation of the actions described in Haddon matrix, their efficiency should be evaluated. The use of more advanced cameras to detect such violations as, for example, crossing into the oncoming lane, can be a good measure to reduce the possibility of accidents on difficult areas. Road barriers separating traffic lanes can also prevent such kinds of accidents (as in European countries).

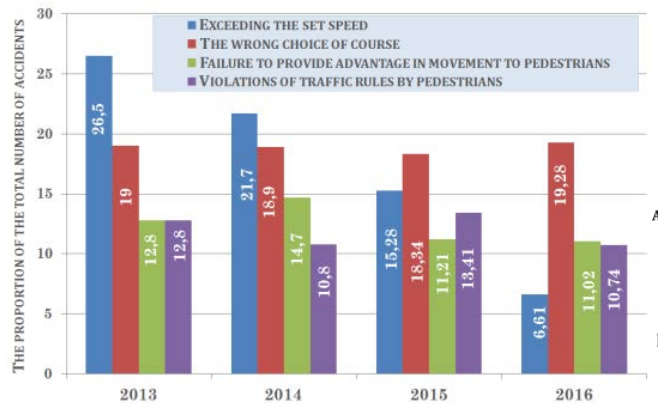

a

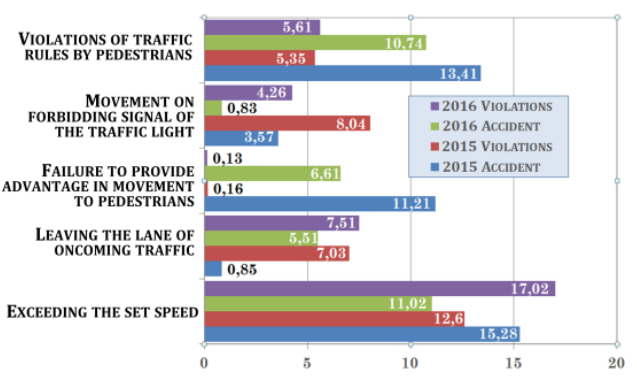

$\mathrm{b}$

Fig. 3. Accidents' analysis a) by causes; b) by relation to violations.

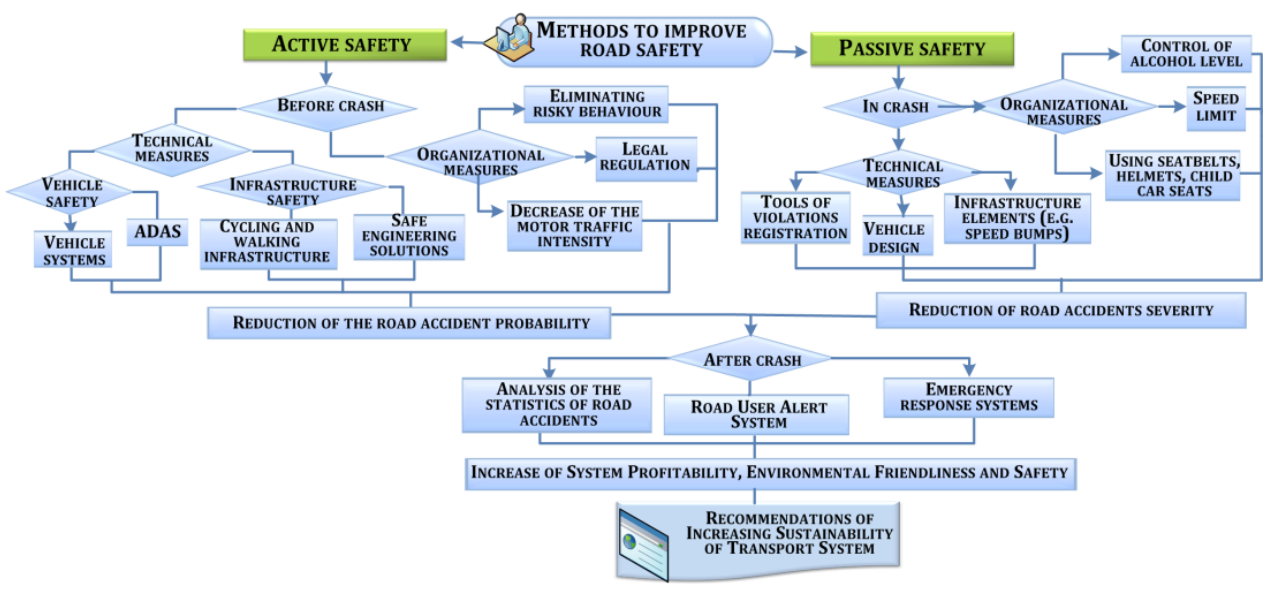

Fig. 4. Ways to improve road safety.

To prevent violations by pedestrians, infrastructure solutions can be used: obstacles to cross the road in the wrong place, overhead pedestrian crossings, underground crosswalks, adjustable crosswalks, etc. It is possible to apply such measures as penalties, as well as establishing cameras in dangerous places. To reduce the number of accidents caused by 
reckless actions of pedestrians, it is necessary to teach the correct behavior on the roads from the early childhood.

If an accident happens, the measures to reduce accidents' severity should be provided. The use of seat-belts and child restraints are understood as a human factor. The vehicle is already designed with all the safety requirements using modern technologies. Infrastructure, in turn, should provide the lowest safety consequence in case of an accident. This can be achieved by such means as barriers, road markings, warning scoreboard, etc.

The actions, which are performed after the accident include the prompt provisioning of assistance to victims of road accidents, traffic recovery, elimination of consequences of the accident and determination of the causes. In Russia, since the January 1, 2017 it is necessary to equip all new vehicles by the emergency response system "ERA-GLONASS" that consists of three elements: navigation and information platform, data network and a communication network. After the accident, the system automatically determines data on the vehicle (brand, model, the VIN number, fuel type), its position, and the magnitude of the overload in the moment of the crash. This system reduces the time of medical aid provisioning and road users' information. All measures are documented in the form of Haddon matrix. Then, the plans of each action's implementation are made (Fig. 5).

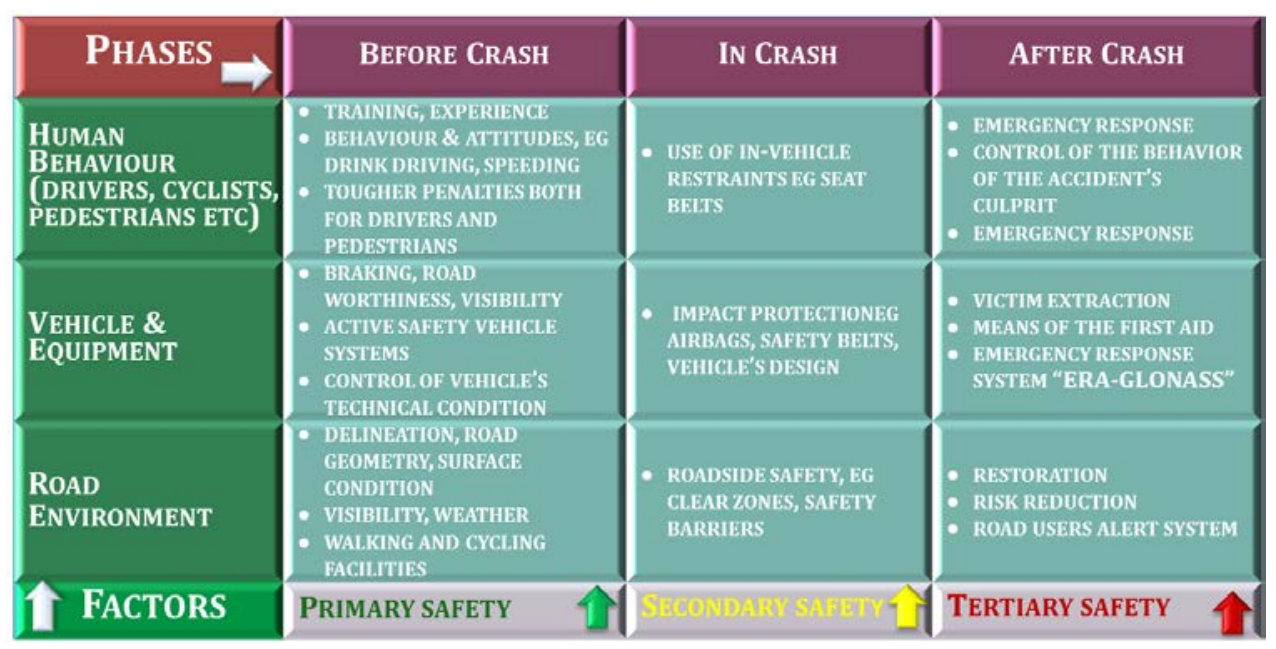

Fig. 5. Haddon's matrix.

\section{Conclusions}

This research is devoted to analysis of traffic violations' causes and their influence on the accidents' possibility. Official statistics of the State Traffic Safety Inspectorate is used as initial information. As a method to develop proposals to improve road safety the Haddon matrix was used. Effectiveness of decisions made should be evaluated based on the accidents statistics before and after interventions.

\section{References}

1. H.P. Baltrenas et al., Research into the impact of speed bumps on particulate matter air pollution. Measurement, 100, p. 62-67 (2017)

2. J. Brown et al. Children and motorcycles: A systematic review of risk factors and interventions. Injury Prevention, (2017) 
3. Federal Target Program "Increase of the road safety for the years 2013-2020", 3th of October 2013, № 864.

4. N.R. Ghadban, et al., A real case-based study exploring influence of human age and gender on drivers' behavior and traffic safety. Advances in Intelligent Systems and Computing, 597, p. 807-816 (2018)

5. Global status report on road safety 2015; URL: http://www.who.int/violence _injury_prevention/road_safety_status/2015/GSRRS2015_Summary_EN_final.pdf.

6. Jr.W. Haddon, Advances in the epidemiology of injuries as a basis for public policy. Public Health Report, 95, p. 411-421 (1980)

7. I. Makarova, et al. Ensuring Sustainability of Public Transport System through Rational Management. Procedia Engineering, 178; p. 137-146 (2017)

8. I. Makarova, et al. Modeling as a method to improve road safety during mass events. Transportation Research Procedia, (20) p. 430-435 (2017)

9. L. Martinussen, et al. How indicative is a self-reported driving behaviour profile of police registered traffic law offences?, Accident Analysis and Prevention, 99, p. 1-5 (2017)

10. H. Mohammed, et al. Simulation Assessment and Theoretical Verification of a New Design for Portable Concrete Barriers. KSCE Journal of Civil Engineering, 21:851, p. 1-12 (2016)

11. P. Penmetsa, et al. Methods to rank traffic rule violations resulting in crashes for allocation of funds, Accident Analysis and Prevention, 99, p.192-201 (2017)

12. N. Rustagi, et al. Applying Haddon Matrix for Evaluation of Road Crash Victims in Delhi, India. Indian Journal of Surgery, May; p. 1-9 (2017)

13. State Traffic Safety Inspectorate; URL: https://www.gibdd.ru/

14. H. Van Lint, et al. Exploring the effects of perception errors and anticipation strategies on traffic accidents - A simulation study. Advances in Intelligent Systems and Computing, 591, p. 249-261 (2018)

15. J.-M. Wang et al. Driving process' analysis and HUD design based on conditional autonomous traffic safety, Lecture Notes in Computer Science, 10287 LNCS, p. 406418. (2017)

16. J. Yang, et al. The Development of Safety Cases for an Autonomous Vehicle: A Comparative Study on Different Methods, SAE Technical Papers, Part F129886; Issue September; URL: http://papers.sae.org/2017-01-2010/ (2017)

17. Y.S. Yoon, et al. A new approach to analysing human-related accidents by combined use of HFACS and activity theory-based method. Cognition, Technology and Work, September, p. 1-25 (2017) 